\title{
Controllable forms for stabilising pole assignment design of generalised bilinear systems ${ }^{1}$
}

\author{
C. James Taylor, Arun Chotai and Keith J. Burnham
}

Bilinear structures are able to represent nonlinear phenomena more accurately than linear models, and thereby help to extend the range of satisfactory control performance. However, closed loop characteristics are typically designed by simulation and stability is not guaranteed. This Letter shows how bilinear systems are a special case of the more general state dependent parameter (SDP) model, which can subsequently be utilised to design stabilising feedback controllers using a special form of nonlinear pole assignment. In order to establish the link, however, an important generalisation of the SDP pole assignment method is developed.

Introduction: Three-term PID control systems are widely used in the process industries. However, the performance of a fixed gain algorithm can be compromised when controlling the system over a wide operating region. Potential solutions to this problem include detuning to enable a wider range of operation, as well as numerous nonlinear design methods, such as gain scheduling and/or the introduction of a bilinear compensator. Bilinear structures are able to represent nonlinear phenomena more accurately than linear models, and thereby extend the range of satisfactory performance. One of the strengths of PID-based bilinear design is the use of classical methods and a relatively straightforward implementation [1-2]. However, closed loop characteristics, such as stability, are typically dependent on empirical investigations.

This Letter shows how bilinear systems are a special case of the more general state dependent parameter (SDP) model [3]. Such models form the basis for stabilising feedback design utilising

\footnotetext{
${ }^{1}$ This paper is a postprint of a paper submitted to and accepted for publication in Electronic Letters and is subject to Institution of Engineering and Technology Copyright. The copy of record is available at IET Digital Library. [Electronic Letters 47, 7, 437-439, 2011]
} 
a special form of pole assignment [4]. In fact, the approach uses the Proportional-Integral-Plus (PIP) framework [5], albeit in a modified form to address the system nonlinearities. Here, algebraic forms of the control gains can usually be derived off-line, to yield a practically useful control algorithm that has a similar degree of complexity to the PID-based bilinear compensator. However, in contrast to [4], the algorithm below is derived entirely in state space form, facilitating a concise stability analysis. Furthermore, in earlier research on SDP pole assignment, the model has been limited to a single element associated with the input, i.e. the system has no open loop zeros. To now address general bilinear models, it is essential to relax this constraint.

Generalized Bilinear Model: Consider the following $n$th order model, with $\tau$ samples time-delay, $m-\tau+1$ parameters associated with the input and $n \times(m-\tau+1)$ additional bilinear terms,

$$
y(k)=\sum_{i=\tau}^{m} b_{i}(k) u(k-i)-\sum_{i=1}^{n} a_{i}(k) y(k-i)+\sum_{i=1}^{n} \sum_{j=\tau}^{m} \eta_{i j} y(k-i) u(k-j)
$$

where $y(k)$ is the output and $u(k)$ the control input. To demonstrate the link with bilinear models $\eta_{i j}$ are constant coefficients, whilst $a_{i}(k)$ and $b_{i}(k)$ are assumed to vary over time in a state dependent manner, i.e. each parameter is expressed as a function of one or more measured variables, not necessarily the input and output. With time invariant $a_{i}(k)=a_{i}$ and $b_{i}(k)=b_{i} \forall k$, equation (1) reduces to the conventional bilinear form [1-2].

Controllable Form: An important concept highlighted by this Letter, is that equation (1) can always be reduced into a controllable SDP model,

$$
y(k)=\tilde{b}(k) u(k-\tau)-\sum_{i=1}^{n} \tilde{a}_{i}(k) y(k-i)
$$

Equation (2) can be derived from (1) or directly estimated [3] in this form. Straightforward algebra shows that the parameters in (2) may be defined in various ways, potentially yielding control systems with different robustness properties. For the purposes of this Letter, however, it is sufficient to note that $b_{i}(k)$ for $i>\tau$ and $\eta_{i j}$ in (1), are associated with $\tilde{a}_{i}(k)$ and $\tilde{b}(k)$ in (2). 
This is best shown by demonstration: consider (1) with $n=m=3, \tau=2$ and time invariant parameters; here, (2) is based on e.g. $\tilde{b}(k)=b_{2}+\sum_{i=1}^{3} \eta_{i 2} y(k-i), \tilde{a}_{1}(k)=a_{1}-\eta_{13} u(k-3)$, $\tilde{a}_{2}(k)=a_{2}-\eta_{23} u(k-3)$ and $\tilde{a}_{3}(k)=a_{3}-\eta_{33} u(k-3)-b_{3} u(k-3) / y(k-3)$.

Nonlinear Pole Assignment: Define a state vector,

$$
\boldsymbol{x}(k)=\left[\begin{array}{lllllllll}
y(k) & y(k-1) & \ldots & y(k-n+1) & u(k-1) & \ldots & u(k-\tau+1) & z(k)
\end{array}\right]
$$

and associated non-minimal state space representation of equation (2),

$$
\boldsymbol{x}(k+1)=\boldsymbol{F}(k) \boldsymbol{x}(k)+\boldsymbol{g}(k) u(k)+\boldsymbol{d} r(k+1) \quad ; \quad y(k)=\boldsymbol{h} \boldsymbol{x}(k)
$$

where $r(k)$ is the command input and $z(k)=z(k-1)+r(k)-y(k)$ is an integral-of-error state variable, introduced to ensure Type 1 servomechanism performance. Here, $\boldsymbol{F}(k), \boldsymbol{g}(k), \boldsymbol{d}$ and $\boldsymbol{h}$ are defined in the obvious way [4-5]. The basic state variable feedback algorithm is,

$$
u(k)=-c(k) \boldsymbol{x}(k)
$$

where $\boldsymbol{c}(k)$ is a vector of scheduled gains. However, for nonlinear pole assignment, a nonsingular transformation matrix $\boldsymbol{T}(k)$ of the state vector is now introduced, such that,

$$
\boldsymbol{T}(k+1) \boldsymbol{x}(k+1)=\boldsymbol{F}(k) \boldsymbol{T}(k) \boldsymbol{x}(k)+\boldsymbol{g}(k) u(k)+\boldsymbol{d} r(k+1)
$$

Substituting $u(k)=-\boldsymbol{c}(k) \boldsymbol{T}(k) \boldsymbol{x}(k)$ into (5) and re-arranging yields the closed loop state equation for $\boldsymbol{x}(k+1)$. Define a matrix $\boldsymbol{D}$ with user specified (arbitrary) eigenvalues $p_{i}$, where $p_{i}$ are the roots of the desired characteristic equation $\lambda^{n}+d_{1} \lambda^{n-1}+\cdots+d_{n+\tau}=0$. If scheduled gains $\boldsymbol{c}(k)$ can be determined so that the closed loop transition matrix equals $\boldsymbol{D} \forall k$, i.e.,

$$
\boldsymbol{T}^{-1}(k+1) \boldsymbol{F}(k) \boldsymbol{T}(k)-\boldsymbol{D}=\boldsymbol{T}^{-1}(k+1) \boldsymbol{g}(k) \boldsymbol{c}(k) \boldsymbol{T}(k)
$$

then the closed loop state equation has the required design eigenvalues. Naturally, such a solution does not exist for arbitrary $\boldsymbol{T}(k)$ and $\boldsymbol{D}$. Furthermore, it is well known that for time varying systems, as here, these eigenvalues do not necessarily determine the performance and stability of the closed loop response. However, now define the following $\boldsymbol{T}(k)$ and $\boldsymbol{D}$ that will 
meet these control goals. For $\tau=1, \boldsymbol{T}(k)=\boldsymbol{T}=\boldsymbol{I}$ is an identity matrix. For $\tau=2$, using $\boldsymbol{a}_{1}(k)=\left[\tilde{a}_{1}(k) \tilde{a}_{2}(k) \cdots \tilde{a}_{n}(k)\right]$,

$$
\boldsymbol{T}(k)=\left[\begin{array}{ccc}
\boldsymbol{I} & 0 & 0 \\
\boldsymbol{a}_{1}(k+1) / \tilde{b}(k+1) & 1 / \tilde{b}(k+1) & 0 \\
\boldsymbol{0} & 0 & 1
\end{array}\right]
$$

where 0 is a row of zeros. For $\tau=3$,

$$
\boldsymbol{T}(k)=\left[\begin{array}{cccc}
\boldsymbol{I} & 0 & 0 & 0 \\
\boldsymbol{a}_{2}(k+2) / \tilde{b}(k+2) & 1 / \tilde{b}(k+2) & \tilde{a}_{1}(k+2) / \tilde{b}(k+2) & 0 \\
\boldsymbol{a}_{1}(k+1) / \tilde{b}(k+1) & 0 & 1 / \tilde{b}(k+1) & 0 \\
\boldsymbol{0} & 0 & 0 & 1
\end{array}\right]
$$

where $\boldsymbol{a}_{2}(k)=\left[\tilde{a}_{2}(k) \tilde{a}_{3}(k) \cdots \tilde{a}_{n}(k) 0\right]$. Following this approach, similar transformations can be developed for $\tau>3$ but are omitted for brevity. For $\tau=1$,

$$
\boldsymbol{D}=\left[\begin{array}{ccccc}
d_{2}+\cdots+d_{n+1} & d_{3}+\cdots+d_{n+1} & \cdots & d_{n+1} & \tilde{d} \\
1 & 0 & \cdots & 0 & 0 \\
0 & 1 & \cdots & 0 & 0 \\
\vdots & \vdots & \ddots & \vdots & \vdots \\
-d_{2}-\cdots-d_{n+1} & -d_{3}-\cdots-d_{n+1} & \cdots & -d_{n+1} & -\tilde{d}-1
\end{array}\right]
$$

in which $\tilde{d}=1+d_{1}+\cdots+d_{n+\tau}$. By contrast, for $\tau>1$,

$$
\boldsymbol{D}=\left[\begin{array}{cccccccc}
0 & 0 & \cdots & 0 & 0 & \cdots & 1 & 0 \\
1 & 0 & \cdots & 0 & 0 & \cdots & 0 & 0 \\
0 & 1 & \cdots & 0 & 0 & \cdots & 0 & 0 \\
\vdots & \vdots & \ddots & \vdots & \vdots & & \vdots & \vdots \\
\tilde{d}_{1} & \tilde{d}_{2} & \cdots & \tilde{d}_{3} & \tilde{d}_{4} & \cdots & \tilde{d}_{5} & \tilde{d} \\
0 & 0 & \cdots & 0 & 1 & \cdots & 0 & 0 \\
\vdots & \vdots & & \vdots & \vdots & \ddots & \vdots & \vdots \\
0 & 0 & \cdots & 0 & 0 & \cdots & -1 & 1
\end{array}\right]
$$

where $\quad \tilde{d}_{1}=d_{n+\tau}+\cdots+d_{n+1} \quad, \quad \tilde{d}_{2}=d_{n+\tau}+\cdots+d_{n+2} \quad, \quad \tilde{d}_{3}=d_{n+\tau} \quad, \quad \tilde{d}_{4}=-1-d_{1} \quad$, $\tilde{d}_{5}=-1-d_{1}-\cdots-d_{\tau-1}$. 
Using these definitions when $\tau>1$, the first $n$ and last $\tau-1$ rows of equation (6) consist only of zeros. By equating the $(n+1)$ th row and solving the resultant set of $n+\tau$ simultaneous equations, the control gains $c(k)$ are obtained. For $\tau=1$, the gains are obtained by equating either the first or final row of (6). In both cases, the control gains in $\boldsymbol{c}(k)$ are identical to those quoted without derivation by [4], who also describe several simulated and practical examples. Furthermore, substitution using the open loop state equation in the manner of Kuo [6], yields a controllability matrix which is non-singular if and only if $\tilde{b}(k) \neq 0$, a result that is particularly easy to check in practice. With time invariant $\tilde{a}_{i}(k)=\tilde{a}_{i}$ and $\tilde{b}(k)=\tilde{b} \forall k$, the control gains in $\boldsymbol{c}(k)=\boldsymbol{c}$ are identical to those obtained using linear PIP methods; in this case, reference [5] describes a more convenient, computational approach for their calculation. The general form of the gains in $\boldsymbol{c}(k)$ are not repeated here. However, the novel state space derivation developed above is ideal for demonstrating the stability of the solution. In fact, assuming no model mismatch, the control law (4), applied to the nonlinear model (1) or its equivalent (3), yields a linear response characterized by the design poles $p_{i}$, as shown below.

Stability: Substituting (4) into (3) yields $\boldsymbol{x}(k+1)=(\boldsymbol{F}(k)-\boldsymbol{g}(k) \boldsymbol{c}(k)) \boldsymbol{x}(k)+\boldsymbol{d} r(k+1)$. When the gains are determined as above, the closed loop transition matrix can always be decomposed into $\boldsymbol{T}(k+1) \boldsymbol{D} \boldsymbol{T}^{-1}(k)$. Hence, pre-multiplying by $\boldsymbol{T}^{-1}(k+1)$ and defining $\tilde{\boldsymbol{x}}(k)=\boldsymbol{T}^{-1}(k) \boldsymbol{x}(k)$ yields $\tilde{\boldsymbol{x}}(k+1)=\boldsymbol{D} \tilde{\boldsymbol{x}}(k)+\boldsymbol{T}^{-1}(k+1) \boldsymbol{d} r(k+1)$. Following a similar approach to Kuo [6], successive substitutions using (7) yields $\tilde{\boldsymbol{x}}(k)=\boldsymbol{D}^{n+\tau} \tilde{\boldsymbol{x}}(k-n-\tau)+\boldsymbol{D}^{n+\tau-1} \boldsymbol{d} r(k-n-\tau+1)+\ldots+\boldsymbol{D}^{0} \boldsymbol{d} r(k)$. The Cayley-Hamilton theorem shows $\boldsymbol{D}^{n+\tau}+d_{1} \boldsymbol{D}^{n+\tau-1}+\ldots+d_{n+\tau} \boldsymbol{I}=\boldsymbol{0}$, hence taking $\tilde{\boldsymbol{x}}(k)+d_{1} \tilde{\boldsymbol{x}}(k-1)+\ldots+d_{n+\tau} \tilde{\boldsymbol{x}}(k-n-\tau)$ and re-arranging yields,

$$
\begin{aligned}
\tilde{\boldsymbol{x}}(k) & =-d_{1} \tilde{\boldsymbol{x}}(k-1)-\ldots-d_{n+\tau} \tilde{\boldsymbol{x}}(k-n-\tau) \\
& +\left(\boldsymbol{D}^{n+\tau-1}+d_{1} \boldsymbol{D}^{n+\tau-2}+\ldots+\boldsymbol{I} d_{n+\tau-1}\right) \boldsymbol{d} r(k-n-\tau+1)+\ldots+\left(\boldsymbol{D}+d_{1} \boldsymbol{I}\right) \boldsymbol{d} r(k-1)+\boldsymbol{d} r(k)
\end{aligned}
$$


Since $y(k)=\boldsymbol{h} \tilde{\boldsymbol{x}}(k)=\boldsymbol{h} \boldsymbol{T}^{-1}(k) \boldsymbol{x}(k)=\boldsymbol{h} \boldsymbol{x}(k)$, it is a trivial matter to obtain the output response. In fact, noting $\boldsymbol{h I d}=\boldsymbol{h D d}=0$ (etc.) and $\boldsymbol{h} \boldsymbol{D}^{n+\tau-1} \boldsymbol{d}=\tilde{d}$, the closed loop response reduces to $y(k)=-d_{1} y(k-1)-\ldots-d_{n+\tau} y(k-n-\tau)+\tilde{d} r(k-\tau)$. Expressed as a linear Transfer Function, the poles are identical to the eigenvalues of $\boldsymbol{D}$ by definition. With design poles inside the unit circle on the complex $z$-plane, closed loop stability clearly follows. $\dagger$

Feasibility: Examination of (6) and $\boldsymbol{T}(k)$ shows that a $\tau$ sample forward shift of the parameters is required. Fortunately, for many systems, the parameters are functions of the delayed input and output signals, hence a forward shift does not cause problems. Significantly, generalisation from the example above, in which $n=m=3$ and $\tau=2$, shows how a bilinear model can always be converted into such a controllable form: here, the SDPs are functions of $u(k-i)$ where $i>\tau$; e.g. $\tilde{a}_{3}(k+2)=a_{3}-\eta_{33} u(k-1)-b_{3} u(k-1) / y(k-1)$. However, predicted values of the output variable are sometimes required. One option is to use $\hat{y}(k+j)=d(k+j)$ where $d(k)$ is the desired response obtained by open loop simulation. In this manner, the analysis above still holds at the design stage, albeit with a robustness penalty in practical applications.

Example: A bilinear model $\dot{y}(t)=\beta_{0} u(t)-\alpha_{0} y(t)+\rho_{0} y(t) u(t)$ has been used to develop control systems for an industrial furnace [2]. For a sampling rate $\Delta t$, one discrete-time bilinear model is $y(k)=b_{1} u(k-1)-a_{1} y(k-1)+\eta_{11} y(k-1) u(k-1)$, in which $b_{1}=\beta_{0}\left(1+a_{1}\right) / \alpha_{0}, a_{1}=-e^{\alpha_{0} \Delta t}$ and $\eta_{11}=\rho_{0} \Delta t$. The controllable SDP model (2) is $y(k)=\left(b_{1}+\eta_{11} y(k-1)\right) u(k-1)-a_{1} y(k-1)$. With a design polynomial $\lambda^{2}+d_{1} \lambda+d_{2}, \quad \boldsymbol{x}(k)=\left[\begin{array}{ll}y(k) & z(k)\end{array}\right]^{T}, \boldsymbol{T}(k)=\boldsymbol{I}$,

$$
\boldsymbol{F}(k)=\left[\begin{array}{cc}
-a_{1} & 0 \\
a_{1} & 1
\end{array}\right] ; \boldsymbol{g}(k)=\left[\begin{array}{c}
b_{1}+\eta_{11} y(k) \\
-b_{1}-\eta_{11} y(k)
\end{array}\right] ; \boldsymbol{D}=\left[\begin{array}{cc}
d_{2} & 1+d_{2}+d_{3} \\
-d_{2} & -d_{1}-d_{2}
\end{array}\right]
$$

and solving the nonlinear pole assignment problem (6), yields $c(k)=\left[\begin{array}{ll}f_{0}(k) & -k_{I}(k)\end{array}\right]$, where $f_{0}(k)=-\left(d_{2}+a_{1}\right) /\left(b_{1}+\eta_{1} y(k)\right)$ and $k_{I}(k)=\left(1+d_{1}+d_{2}\right) /\left(b_{1}+\eta_{1} y(k)\right)$ are the scheduled proportional and integral gains respectively. For this example, an equivalent fixed gain PIP or PID design typically yields an unstable response (away from the design operating level). By 
contrast, SDP-PIP controllers applied to the discrete-time bilinear model, will always yield an exact match to the designed for response based on the chosen pole positions.

When the SDP controller is now applied to the continuous-time bilinear model quoted above, it still yields a response almost identical to the required solution, even in the case of dead-beat design, in which the closed loop poles are placed at the origin of the complex z-plane and the output reaches the set point after $\tau$ sampling intervals (of course, this is not usually recommended for practical applications). For this example, a PIP controller [5] combined with a bilinear compensator [2] yields similar simulation results to the SDP-PIP approach. In fact, the authors are presently investigating the relative performance and robustness of SDP and bilinear compensator-based control systems, applied to a range of examples with realistic levels of model uncertainty. However, this is beyond the scope of the present Letter.

Conclusions: This Letter has shown how a bilinear model with open loop zeros may be converted into a controllable SDP form, hence allowing for a significant generalisation of a previously developed nonlinear pole assignment algorithm. Furthermore, a new demonstration of the veracity of the algorithm has been developed, in which the calculation of the control gains and the stability proof are now presented in a unified state space form, potentially allowing for future extension of these methods to the multivariable and optimal design cases.

\section{References}

1 Dunoyer, A., Balmer, L., Burnham, K.J. and James, D.J.G.: 'On the discretization of singleinput single-output bilinear systems', Int. J. Control, 1994, 68, 2, pp. 361-372.

2 Martineau, S., Burnham, K.J., Haas, O.C.L., Andrews, G. and Heeley, A.: 'Four-term bilinear PID controller applied to an industrial furnace', Control Eng. Practice, 2004, 12, pp. 457-464.

3 Sadeghi, J., Tych, W., Chotai, A. and Young, P.C. (2010) Multi-state dependent parameter model identification and estimation for nonlinear dynamic systems, Electronics Letters, 46, 18. 
4 Taylor, C.J., Chotai, A. and Young, P.C.: 'Nonlinear control by input-output state variable feedback pole assignment', Int. J. Control, 2009, 82, 6, pp. 1029-1044.

5 Young, P.C., Behzadi, M.A., Wang, C.L. and Chotai, A.: 'Direct digital and adaptive control by input-output, state variable feedback pole assignment', Int. J. Control, 1987, 46, 1867-1881.

6 Kuo, B.C.: 'Digital Control Systems', Second Edition, Oxford University Press, 1997.

\section{Author Affiliations}

C. James Taylor, Engineering Department, Lancaster University, Lancaster LA1 4YR

Arun Chotai, LEC, Lancaster University, Lancaster LA1 4YQ

Keith Burnham, CTAC, Coventry University, CV1 5FB

Email: c.taylor@lancaster.ac.uk 